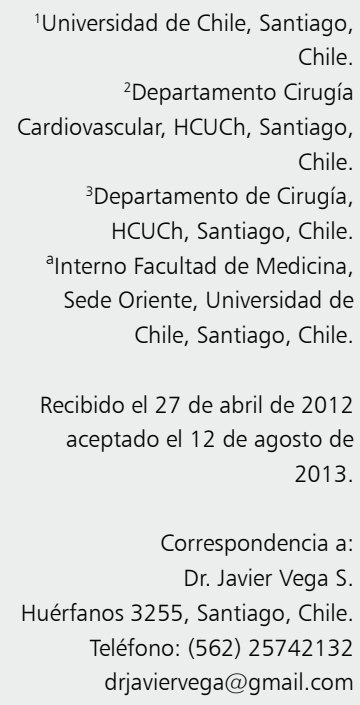

Correspondencia a: Dr. Javier Vega S. Huérfanos 3255, Santiago, Chile. Teléfono: (562) 25742132 drjaviervega@gmail.com

\section{Síndrome aórtico agudo. Revisión de la literatura y actualización del tema}

\author{
JAVIER VEGA S. ${ }^{1}$, JAIME ZAMORANO G. ${ }^{2}$, \\ NICOLÁS PEREIRA C. ${ }^{3}$, ALFONSO GALLEGUILLOS G. ${ }^{a}$
}

\section{Acute aortic syndrome}

Acute aortic syndrome (AAS) is a term that describes interrelated aortic emergencies with similar clinical characteristics and challenges. These are aortic dissection (AD), intramural hematoma (IH), and penetrating atherosclerotic ulcer (PAU). The incidence of AAS is three cases per 100.000 persons per year. Diverse genetic disorders and acquired conditions have been related to the pathogenesis of this disease. Clinical features of patients with any of the three conditions comprising AAS are very similar. A high degree of clinical suspicion and imaging studies are necessary for an accurate diagnosis. Prognosis is clearly related to underlying diagnosis and appropriate surgical repair, in the case of proximal involvement of the aorta. Involvement of distal segments of the aorta may require medical or endovascular therapy according to the presence of complications. After hospital discharge, patients require lifelong follow-up.

(Rev Med Chile 2014; 142: 344-352)

Key words: Aortic arch syndromes; Aortic dissection; Atherosclerosis.
$\mathrm{E}$ 1 sindrome aórtico agudo (SAA) hace alusión a diversas enfermedades con características clínicas similares y elevado riesgo vital $^{1}$. Las enfermedades involucradas son tres: la disección aórtica (DA), el hematoma intramural (HI) y la úlcera aterosclerótica penetrante (UAP $)^{2}$.

\section{Epidemiología}

La incidencia del SAA es de 3 casos por cada 100.000 habitantes al año. De todos los pacientes que debutan con un SAA, $80 \%$ corresponde a DA, $15 \%$ a $\mathrm{HI}$ y $5 \%$ a $\mathrm{UAP}^{3}$.

En relación a los casos de DA, una revisión de 464 pacientes del International Registry of Acute Aortic Dissection (IRAD) demostró que dos tercios de los pacientes eran varones, con una edad media de 63 años.

Aunque las mujeres suelen resultar afectadas con menor frecuencia por la DA aguda, su edad es significativamente mayor que la de los varones, con una media de 67 años 5 .

El 20\% de los pacientes afectados por una DA fallece antes de llegar al hospital, 30\% durante la hospitalización y $20 \%$ en los 10 años posteriores al evento ${ }^{6}$.

Se ha demostrado una asociación entre la DA, el ritmo circadiano y las variaciones estacionales. La mayor frecuencia se perfila entre las 08:00 y 09:00 h y durante los meses de invierno ${ }^{7}$. Esto estaría relacionado con el ritmo circadiano de la presión arterial, que también presenta un aumento durante las mañanas ${ }^{8}$.

La presentación clínica, así como los factores predisponentes del HI no difieren de la DA clásica ${ }^{9}$.

La UAP se presenta típicamente en individuos mayores (edad media de 73 años), hipertensos crónicos, hiperlipidémicos y tiene una leve predilección por el género masculino $(60 \% \text { de los casos })^{10}$.

\section{Fisiopatología}

El SAA se presenta cuando una solución de continuidad o úlcera permite el paso de sangre desde el lumen aórtico a la capa media; o se ge- 
nera una rotura de los "vasa vasorum" dentro de dicha capa. Con el paso de las horas, esta sangre (sumada al efecto de la presión arterial sistémica) es capaz de desencadenar una disección o ruptura aórtica $^{1-11}$ (Figura 1).

Los trastornos, tanto adquiridos como genéticos, comparten una vía final común que da lugar a la disrupción de la íntima. Todos los mecanismos que debilitan la capa media de la aorta, asociados a una mayor tensión en la pared vascular, pueden inducir la dilatación de ésta y la formación de un aneurisma, o bien, causar un HI, DA o rotura arterial. El factor de riesgo más frecuente en la DA es la hipertensión arterial (HTA), que expone de manera crónica a la aorta a altas presiones que dan lugar a un engrosamiento de la íntima, fibrosis, calcificación y depósito extracelular de ácidos grasos; la matriz extracelular experimenta degradación acelerada, apoptosis y elastolisis con disrupción final de la íntima ${ }^{12-15}$ (Tabla 1).

La DA, generada por esta disrupción de la íntima, habitualmente es precedida por degeneración o necrosis quística de la media ${ }^{12}$. La sangre es capaz de atravesar esta anomalía anatómica y separar la capa íntima de la media o adventicia, creando un lumen falso. La propagación de la disección puede ser en sentido anterógrado o retrógrado (en relación a la solución de continuidad) y causar una ruptura aórtica, insuficiencia cardíaca o sindromes de mala perfusión ${ }^{17-19}$.

La insuficiencia cardíaca es generada por insuficiencia aórtica, infarto agudo de miocardio (IAM) y taponamiento cardíaco. La insuficiencia aórtica está explicada por la dilatación del anillo y la raíz aórtica, depresión de una valva, prolapso del flap (colgajo) y torsión del anillo ${ }^{20,21}$. El IAM suele ubicarse en la pared cardíaca inferior y se debe a la disección retrógrada que compromete el ostium de la arteria coronaria derecha ${ }^{22}$.

El efecto de la disección en la perfusión de las distintas ramas arteriales originadas de la aorta es complejo, ya que depende del grado de colapso del lumen verdadero, la relación de la rama arterial con el flap intimal y del nivel de perfusión aportado por el lumen verdadero o falso. Sin embargo, la isquemia visceral es más frecuente cuando la disección afecta la circunferencia completa de la aorta y hay una presión elevada en el lumen falso ${ }^{23-25}$.

El HI se origina en la rotura de los "vasa vasorum" dentro de la capa media. Éste puede generar un pseudoaneurisma, una ruptura y $\mathrm{DA}^{26-28}$.

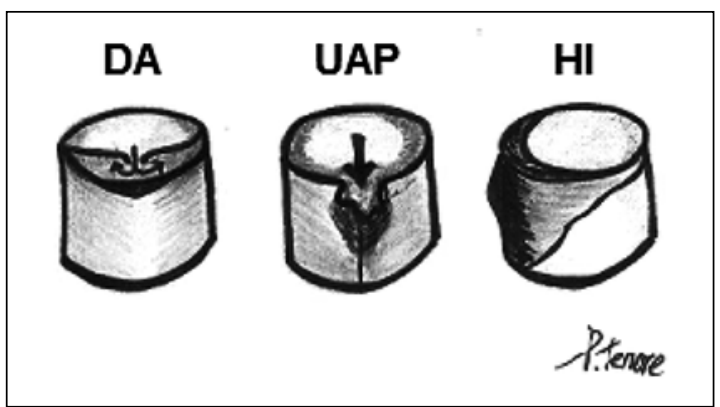

Figura 1. Figura esquemática de las enfermedades involucradas en el síndrome aórtico agudo. DA, disección aórtica; UAP, úlcera aterosclerótica penetrante; $\mathrm{HI}$, hematoma intramural.

Tabla 1. Patologías asociadas en 36 pacientes tratados por DA torácica tipo B entre octubre de 2001 y agosto de 2007, según Mertens et al ${ }^{16}$

\begin{tabular}{|lc|}
\hline Hipertensión arterial & $77,8 \%$ \\
\hline Tabaquismo & $38,9 \%$ \\
\hline Dislipidemia & $25,0 \%$ \\
\hline Obesidad & $11,1 \%$ \\
\hline Insuficiencia renal crónica & $8,3 \%$ \\
\hline Enfermedad de Marfán & $8,3 \%$ \\
\hline Cardiopatía coronaria & $5,6 \%$ \\
\hline
\end{tabular}

La UAP corresponde a la ulceración profunda de una placa aórtica aterosclerótica desde la lámina elástica interna a la capa media. Suele provocar un $\mathrm{HI}^{21} \mathrm{y}$ en determinadas ocasiones complicarse con la formación de un aneurisma, pseudoaneurisma, disección y ruptura aórtica².

\section{Clasificación del SAA}

Hoy en día la clasificación más utilizada es la de Stanford (Figura 2), que hace alusión exclusivamente a la DA. La razón de esto es que entrega información de la extensión de la disección en vez del proceso fisiopatológico involucrado, información que resulta indispensable para decidir el tipo de manejo ${ }^{29}$.

Adicionalmente, dependiendo del tiempo de evolución desde la instalación de los síntomas, es posible diferenciar una DA aguda de una crónica, si éste es menor o mayor a 2 semanas, respectivamente $^{21}$. 


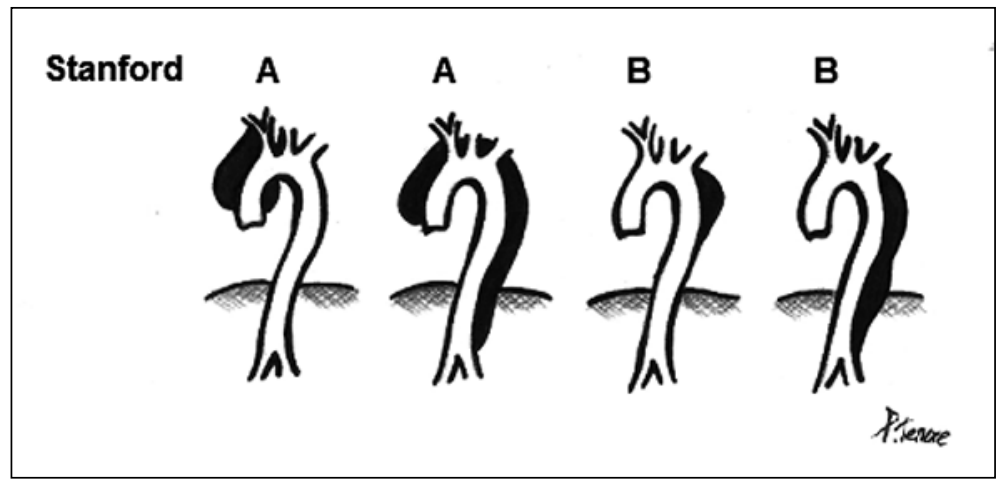

Figura 2. Clasificación de la DA según Stanford. Stanford A: compromete aorta ascendente. Stanford B: no compromete aorta ascendente.
Al igual que en la disección clásica, se distinguen, según la clasificación de Stanford, 2 tipos de HI: tipo A, siempre que haya afectación de la aorta ascendente y tipo B, cuando la aorta ascendente está respetada ${ }^{30}$.

\section{Manifestaciones clínicas}

Los pacientes afectados por un SAA poseen manifestaciones clínicas similares, independiente de la entidad patológica de base; ya sea una DA, $\mathrm{UAP}$ o un $\mathrm{HI}^{1}$.

El dolor es el síntoma cardinal y su reconocimiento es de vital importancia, ya que el SAA es la condición fatal más frecuente de dolor toráxico. Se localiza en la región anterior del tórax, cuello, garganta y mandíbula. También se le describe en la región dorsal siguiendo hacia caudal hasta la región abdominal. Se caracteriza por ser severamente intenso y los pacientes lo describen como un dolor de inicio súbito, punzante y desgarrante ${ }^{4}$. En reiteradas ocasiones suele confundirse con un síndrome coronario agudo (SCA). La diferencia semiológica radica en que el SAA comienza con intensidad máxima y va declinando con el correr de las horas, en cambio el dolor del SCA es leve al comienzo y la intensidad aumenta después de algunos minutos ${ }^{31}$.

La Tabla 2 describe los síntomas más frecuentes objetivados en los pacientes con DA aguda. Agrupando los síntomas, el médico podría orientarse clínicamente al tipo de DA que se está enfrentando. Un paciente que presenta dolor torácico severo (sin irradiación al dorso), síncope, hipotensión arterial, taponamiento cardíaco, insuficiencia aórtica, IAM o shock cardiogénico; es más probable que sea portador de una DA tipo A. En contraste con el portador de una DA tipo $B$ que refiere dolor torácico severo, irradiado a la región interescapular, de tipo migratorio y al examen físico presenta cifras tensionales elevadas, claudicación o diferencia en la amplitud del pulso entre las extremidades superiores e inferiores ${ }^{1}$. A pesar de esto, alrededor de $10 \%$ de los pacientes afectados por una DA no se queja de dolor 4 .

El HI puede generar dolor toráxico con o sin irradiación al dorso, HTA y en casos aislados taponamiento cardíaco ${ }^{1}$.

La UAP afecta principalmente a la aorta descendente y en etapas iniciales los pacientes suelen ser asintomáticos, pero cuando el proceso es más avanzado pueden referir dolor de baja intensidad en la región dorsolumbar o abdominal. En los

Tabla 2. Síntomas y signos en los pacientes portadores de una DA aguda ${ }^{4}$

\begin{tabular}{|ll|}
\hline Dolor severo o el "peor de la vida" & $90 \%$ \\
\hline Inicio abrupto del dolor & $90 \%$ \\
\hline Dolor toráxico con o sin irradiación al dorso & $85 \%$ \\
Hipertensión (PAS $\geq 150 \mathrm{mmHg}$ ) & $49 \%$ \\
Insuficiencia aórtica & $32 \%$ \\
Dolor abdominal & $30 \%$ \\
Pulso parvus et tardus & $27 \%$ \\
Dolor migratorio & $19 \%$ \\
Hipotensión (PAS < $100 \mathrm{mmHg}$ ), shock o & $18 \%$ \\
taponamiento cardíaco & \\
\hline Déficit neurológico focal & $12 \%$ \\
\hline
\end{tabular}

PAS, presión arterial sistólica; Parvus et tardus, pulso disminuido en amplitud y de ascenso lento. 
casos más severos en que hay ruptura aórtica, el shock hipovolémico es evidente ${ }^{1,2}$.

\section{Historia natural y pronóstico}

En relación a la DA tipo A, la mortalidad es mayor en aquellos pacientes que presentan complicaciones como taponamiento cardíaco, IAM y mal perfusión cerebral ${ }^{17,32-34}$. Otros predictores de mal pronóstico son la edad $>70$ años, hipotensión, falla renal aguda (FRA) y déficit de pulso ${ }^{35}$. Una DA iatrogénica conlleva una mortalidad mayor que una DA no iatrogénica (35 vs $24 \%)^{36}$. En ausencia de tratamiento quirúrgico inmediato, el tratamiento médico presenta una mortalidad promedio a las $24 \mathrm{~h}$ de $20 \%$, a las $48 \mathrm{~h}$ de $30 \%$, a los 7 días de $40 \%$ y a los 30 días de $50 \%$. Con tratamiento quirúrgico la mortalidad a las $24 \mathrm{~h}$ disminuye a $10 \%$, a los 7 días a $13 \%$ y a los 30 días a $20 \%{ }^{4}$. Las causas de muerte más frecuentes son la ruptura aórtica con el taponamiento cardíaco $(41,6 \%)$ y la isquemia visceral $(13,9 \%)^{4}$.

La mortalidad a los 30 días de los pacientes con
DA tipo B no complicada es cercana al $10 \%{ }^{4}$. Por el contrario, en los pacientes con mala perfusión visceral o periférica, insuficiencia renal o signos de rotura contenida, la mortalidad supera $30 \%{ }^{37,38}$.

El HI tiene una mortalidad a los 3 meses de $19 \%$ y los principales predictores independientes de mortalidad son el diámetro aórtico $(>50 \mathrm{~mm})$ y la afectación de la aorta ascendente ${ }^{5}$.

En relación a la UAP, el riesgo de complicarse con una ruptura es de $38 \%$ si se presenta como un SAA. La UAP asintomática (descubierta por imágenes) presenta tasas de ruptura y progresión mucho menores ${ }^{11}$.

\section{Diagnóstico}

Para establecer un diagnóstico rápido y certero, es necesario mantener un alto índice de sospecha y llevar a cabo una evaluación básica, que incluya un ECG y radiografía de tórax. Luego se debe completar el estudio con otras técnicas imagenológicas (Figura 3).

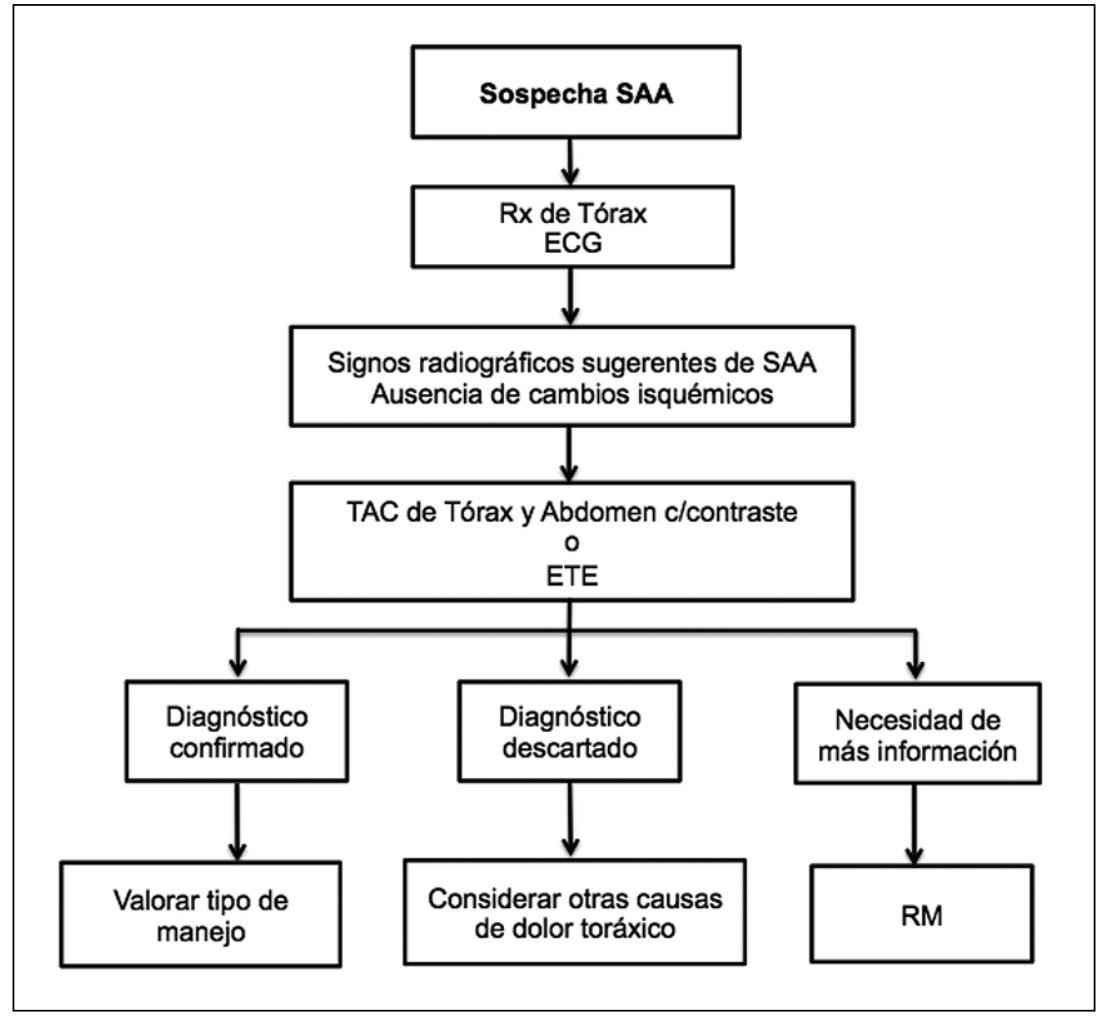

Figura 3. Algoritmo diagnóstico en el manejo de los pacientes con sospecha de SAA. SAA, síndrome aórtico agudo; $\mathrm{Rx}$, radiografía; ECG, electrocardiograma; ETE, ecocardiografía transesofágica; TAC, tomografía axial computarizada; RM, resonancia magnética. 

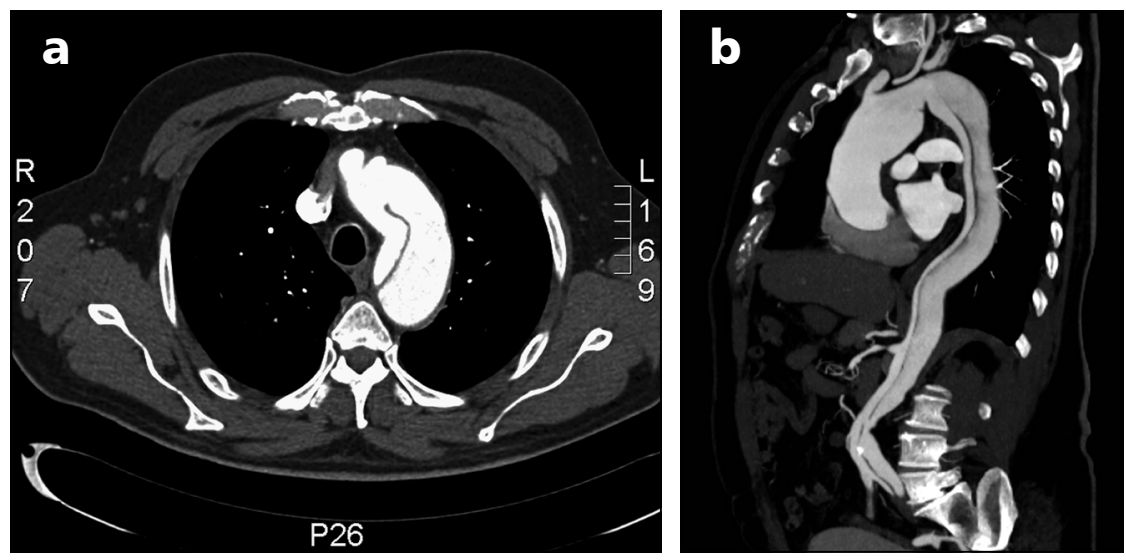

Figura 4. Tomografía computada, corte axial (a) y sagital (b). Se observa una DA tipo $A$, que se origina desde la aorta ascendente, comprometiendo aorta toráxica descendente y abdominal, hasta el origen de las arterias ilíacas. Se distingue claramente el flap intimal (flecha).

Aunque el ECG no apoya el diagnóstico de SAA, permite diferenciar entre una DA y un SCA, puesto que el cuadro clínico puede ser muy parecido $^{20}$.

Si bien la radiografía de tórax, adecuadamente realizada e interpretada por expertos, tiene una alta precisión diagnóstica, la realidad es que en menos de $30 \%$ de los pacientes, existen signos inequívocos de DA. Sin embargo, se acepta que en el proceso diagnóstico es esencial contar con una radiografía de tórax porque no sólo puede ofrecer signos compatibles y apoyar la necesidad de realizar otra prueba de imagen para confirmar el diagnóstico, sino que también permite identificar otras causas de dolor toráxico ${ }^{39}$. Los signos radiográficos que sugieren DA son: borramiento o ensanchamiento del botón aórtico, derrame pleural (generalmente izquierdo), desviación de la tráquea, distancia mayor de $6 \mathrm{~mm}$ entre una calcificación intimal y el contorno externo de la pared aórtica, ensanchamiento mediastínico, ensanchamiento de la aorta ascendente o descendente y ensanchamiento de la línea paraespinal ${ }^{40}$.

Una vez obtenida una evaluación básica concordante con la sospecha clínica, se recomienda la utilización de una técnica imagenológica como la tomografía computarizada (TAC) de tórax y abdomen con contraste o el ecocardiograma transesofágico (ETE), dependiendo de la disponibilidad del centro ${ }^{41}$.

La ETE ha cambiado radicalmente la actitud diagnóstica en la DA. El esófago está en íntimo contacto con la aorta, por lo que su estudio es muy completo. La gran limitación es la dificultad para estudiar la porción más alta de la aorta ascendente por la interposición de la tráquea y el bronquio principal izquierdo, aunque es muy raro que una disección se localice exclusivamente en esa región ${ }^{20}$. La sensibilidad, la especificidad y los valores predictivos están por encima de $95 \%{ }^{42}$.

La TAC tiene una especificidad que va del 87 al $100 \%$ y una sensibilidad del 96 al 100\% para el diagnóstico de $\mathrm{DA}^{43}$. Esta última disminuye a menos de $80 \%$ cuando la disección afecta a la aorta ascendente ${ }^{44}$. En contexto de urgencia, es probablemente el examen que aporta mayor información y responde a la mayoría de los objetivos, evaluando además, las ramas viscerales de la aorta abdominal $^{43}$ (Figura 4).

La RM se perfila como el examen con mayor sensibilidad y especificidad ${ }^{45}$, pero no se recomienda en el estudio inicial por el tiempo requerido para obtener las imágenes y la disponibilidad excepcional en los servicios de urgencia ${ }^{21}$.

\section{Tratamiento}

El manejo inicial del SAA tiene como objetivo principal, disminuir el shear stress sobre la pared aórtica, mediante el control de la presión arterial y el $\mathrm{d} P / \mathrm{d} t^{1,21}$.

La primera línea de tratamiento es con beta bloquedores endovenosos (ej: labetalol, propanolol). Para los pacientes en que dichos fármacos están contraindicados (ej: asmáticos), se prefiere el uso de bloqueadores de canales de calcio endovenosos (ej: verpamilo, diltiazem $)^{29,46}$. El objetivo es mantener una PAS que fluctúe entre 100 y 120 $\mathrm{mmHg}$ y un rango de frecuencia cardíaca entre 60 y $80 \mathrm{lpm}^{47}$. 
Además se recomienda el uso de un analgésico opioide (ej: morfina) para atenuar la descarga simpática de catecolaminas generada por el dolor, que provoca hipertensión y taquicardia ${ }^{1,47}$.

La DA tipo A es una urgencia quirúrgica y por ende, una vez aclarado el diagnóstico, debe ser sometida a tratamiento lo antes posible. El objetivo es la prevención de la ruptura o la aparación de un derrame pericárdico, que puede conducir al taponamiento cardíaco y la muerte ${ }^{47}$. El procedimiento consiste en resecar la aorta ascendente y el arco aórtico (este último sólo si está afectado) y reemplazarlo por un injerto protésico. También es necesaria la reparación o reemplazo de la válvula aórtica, en los casos en que se presentan cambios patológicos en la raíz aórtica o en la válvula propiamente tal. Además del reemplazo valvular, dependiendo de la anatomía y el estado del ostium de las coronarias, en ocasiones es mandatorio la reconstrucción de estas últimas ${ }^{48}$. Según un reporte de la IRAD el promedio de mortalidad intrahospitalaria luego un tratamiento quirúrgico convencional de una DA tipo A fue de $24 \%{ }^{49}$. En nuestro país, Selman et al, mediante un análisis retrospectivo, registraron una mortalidad operatoria de $27 \%$, en 85 pacientes con DA tipo $\mathrm{A}^{41}$. Promedios similares se han publicado en otros reportes y alrededor de $55 \%$ de los pacientes retorna a su vida independiente ${ }^{50-53}$.

Con respecto a la DA tipo B, el tratamiento va a depender de la presencia de complicaciones.

En la DA tipo B no complicada el tratamiento médico se perfila como el gold standard ${ }^{1}$, teniendo en consideración que el 14 a $67 \%$ de los pacientes que son sometidos a cirugía, fallece o adquiere un daño irreversible a nivel medular ${ }^{4,19}$.

Las complicaciones de la DA tipo B pueden dividirse en dos grupos, el primero es la amenaza de ruptura aórtica o ruptura franca, que se manifiesta por dolor persistente, aparición de derrame pleural denso o compromiso hemodinámico por hemorragia. El segundo grupo comprende complicaciones de tipo isquémico. Éstas corresponden a la isquemia localizada de un órgano o extremidad y a la hipertensión intratable por un fenómeno de pseudocoartación dinámica o permanente ${ }^{16}$. En ambos grupos el procedimiento endovascular puede resolver en forma expedita y poco invasiva ${ }^{54,55}$ un problema que la cirugía abierta requeriría de extensos y cruentos procedimientos con una alta morbimortalidad asociada ${ }^{56,57}$. La intervención consiste en desplegar una endoprótesis (stent) en el lumen verdadero para impedir la comunicación más proximal entre éste y el lumen falso. De esta manera se induce una trombosis, en relación con la endoprótesis, del lumen falso a nivel torácico ${ }^{16}$, disminuyendo la dilatación progresiva y favoreciendo un remodelado adecuado ${ }^{46}$. El concepto de PETTICOAT (Provisional Extension to Induce Complete Attachment) considera la idea de extender la endoprótesis distalmente mediante el uso de stents de metal de celdas abiertas, hasta que la mal perfusión distal se corrija ${ }^{58}$. Por lo general, no se necesitan maniobras de fenestración aórtica o revascularización con ramas, que actualmente están casi obsoletas ${ }^{59}$.

Mediante una intervención endovascular exitosa, la mortalidad disminuye a menos de $10 \%$ y el riesgo de paraplejia a menos de $3 \%{ }^{46}$.

$\mathrm{Al}$ igual que sucede en las DA, en los pacientes con HI tipo A se aconseja la cirugía, y en los portadores de un HI tipo B, el tratamiento médico inicial $^{47}$.

Debido al gran potencial de progresión y complicaciones de la UAP ubicada en la aorta ascendente, se recomienda el tratamiento quirúrgico. Por otro lado, en los casos en que la aorta descendente es la afectada, el tratamiento médico conservador es aceptado. Sin embargo, si hay signos de progresión, como dolor intratable, aumento del grosor de la pared aórtica, diámetro de la UAP mayor a $20 \mathrm{~mm}$, profundidad de la UAP mayor a $10 \mathrm{~mm}, \mathrm{HI}$, sangre extra adventicial y aumento de la efusión pleural, se recomienda el tratamiento endovascular ${ }^{2}$.

\section{Seguimiento}

La sobrevida a 10 años de los pacientes que son hospitalizados por un SAA varía de 30 a $60 \%$ en los diferentes estudios ${ }^{18,50,60}$.

El estado de la aorta debe evaluarse con TAC al $1^{\text {er }}, 3^{\text {er }}, 6^{\text {to }}, 12^{\text {vo }}$ mes y posteriormente una vez por año. Este examen permite detectar los cambios que se generan en la aorta, tanto precoces como tardíos, después de una intervención quirúrgica o un tratamiento médico ${ }^{61}$, como un eventual aumento del diámetro aórtico, signos de formación aneurismática y hemorragias en los sitios de anastomosis o colocación de stents.

Además del control imagenológico, se deben 
tratar los factores de riesgo como la HTA (idealmente con beta bloqueadores), evitar los ejercicios isométricos y las actividades que puedan provocar un trauma toráxico ${ }^{21,29}$. El paciente debe ser educado acerca de su condición y conocer los síntomas de alerta que deben llevarlo a consultar ${ }^{62,63}$.

\section{Conclusión}

Teniendo en cuenta que el SAA es un grupo de enfermedades que involucra un elevado riesgo vital, es deber del médico sospecharlo, al presentarse los primeros indicios. Así, mediante una evaluación básica y un apoyo imagenológico adecuado, es posible llegar a un diagnóstico de manera rápida y certera, que permite ofrecer la mejor alternativa de tratamiento.

Hoy en día, el mayor entendimiento de la fisiopatología involucrada, sumado al avance tecnológico, permite incorporar nuevas estrategias terapéuticas, como son los procedimientos endovasculares, que resultan ser menos invasivos y más efectivos. Todo esto, asociado al entusiasmo científico por comprender la historia natural de la enfermedad, logrará con el paso de los años, disminuir su morbimortalidad.

\section{Referencias}

1. Nienaber C, Powell J. Management of acute aortic syndromes. Eur Heart J 2012; 33: 26-35.

2. Singhal P, Lin Z. Penetrating atheromatous ulcer of ascending aorta: a case report and review of literature. Heart Lung Circ 2008; 17 (5): 380-2.

3. Evangelista M. Historia natural y tratamiento del síndrome aórtico agudo. Rev Esp Cardiol 2004; 57 (7): 667-79.

4. Hagan P, Nienaber C, Isselbacher E, Bruckman D, Karavite $\mathrm{D}$, Evangelista $\mathrm{A}$, et al. The International Registry of Acute Aortic Dissection (IRAD). New insights into an old disease. JAMA 2000; 283: 897-903.

5. Nienaber C, Fattori R, Mehta R, Richartz B, Evangelista A, Petzsch M, et al. Gender-related differences in acute aortic dissection. Circulation 2004; 109: 3014-21.

6. Olsson C, Thelin S, Stahle E, Ekbom A, Granath F. Thoracic aortic aneurysm and dissection: increasing prevalence and improved outcomes reported in a nationwide population-based study of more than 14000 cases from 1987 to 2002. Circulation 2006; 114: 2611-8.

7. Mehta R, Manfredini R, Hassan F, Sechten U, Bossone E, Oh J, et al. Chronobiological patterns of acute aortic dissection. Circulation 2002; 106: 1110-5.

8. Craig M, Bishop C, Raftery E. Circadian variation of blood pressure. Lancet 1978; 311: 795-7.

9. Gil J, Caralps J, Padró J, Cámara M, Montiel J, Arís A. Hematoma intramurales en aorta ascendente. Rev Esp Cardiol 1998; 51: 69-71.

10. Nathan D, Boonn W, Lai E, Wang G, Desai N, Woo E, et al. Presentation, complications, and natural history of penetrating atherosclerotic ulcer disease. J Vasc Surg 2012; 55: 10-5.

11. Vilacosta I, Aragoncillo P, Cañadas V, San Román J, Ferreirós J, Rodríguez E. Acute aortic syndrome: a new look at an old conundrum. Heart 2009; 95: 1130-9.

12. Larson E, Edwards W. Risk factors for aortic dissection: a necropsy study of 161 cases. Am J Cardiol 1984; 53: 849-55.

13. Reed D, Reed C, Stemmermann G, Hayashi T. Are aortic aneurysms caused by atherosclerosis? Circulation 1992; 85: 205-11.

14. Stefanadis C, Karayannacos P, Boudoulas H, Stratos C, Vlachopoulos C, Dotas I, et al. Medial necrosis and acute alterations in aortic distensibility following removal of the vasa vasorum of canine ascending aorta. Cardiovasc Res 1993; 27: 951-6.

15. Von Kodolitsch Y, Aydin M, Koschyk D, Loose R, Schalwat I, Karck M, et al. Predictors of aneurysmal formation after surgical correction of aortic coarctation. J Am Coll Cardiol 2002; 39: 617-24.

16. Mertens R, Arriagada I, Valdés F, Krämer A, Mariné L, Bergoeing $\mathrm{M}$, et al. Tratamiento endovascular de la disección aórtica tipo B mediante endoprótesis. Rev Med Chile 2008; 136: 1431-8.

17. Suzuki T, Mehta R, Ince H, Nagai R, Sakomura Y, Weber $F$, et al. Clinical profiles and outcomes of acute type $B$ aortic dissection in the current era: lessons from the International Registry of Aortic Dissection (IRAD). Circulation 2003; 108: II312-7.

18. Svensson L, Kouchoukos N, Miller D, Bavaria J, Coselli J, Curi M, et al. Expert consensus document on the treatment of descending thoracic aortic disease using endovascular stent-grafts. Ann Thorac Surg 2008; 85: S1-S41.

19. Nienaber C, Zannetti S, Barbieri B, Kische S, Schareck W, Rehders T. Investigation of stent grafts in patients with type B aortic dissection: design of the INSTEAD trial: a prospective, multicenter, European randomized trial. Am Heart J 2005; 149: 592-9.

20. Zamorano J, Mayordomo J, Evangelista A, San Román J, Bañuelos C, Gil Aguado M. Guías de práctica clínica de la Sociedad Española de Cardiología en enfermedades de la aorta. Rev Esp Cardiol 2000; 53: 531-41. 
21. Braverman A. Aortic dissection: prompt diagnosis and emergency treatment are critical. Cleveland Clin J Med 2011; 78 (10): 685-96.

22. Contreras E, Zuluaga S, Gómez J, Ocampo V, Urrea C. Disección aórtica: estado actual. Rev Costarric Cardiol 2009; 11 (1): 19-27.

23. Williams D, LePage M, Lee D. The dissected aorta: part I. Early anatomic changes in an in vitro model. Radiology 1997; 203: 23-31.

24. Chung J, Elkins C, Sakai T, Kato N, Vestring T, Semba $\mathrm{C}$, et al. True-lumen collapse in aortic dissection: part II. Evaluation of treatment methods in phantoms with pulsatile flow. Radiology 2000; 214: 99-106.

25. Williams D, Lee D, Hamilton B, Marx M, Narasimham D, Kazanjian S, et al. The dissected aorta: part III. Anatomy and radiologic diagnosis of branch-vessel compromise. Radiology 1997; 203: 37-44.

26. Hiratzka L, Bakris G, Beckman J, Bersin R, Carr V, Casey D, et al. ACCF/AHA/AATS/ACR/ASA/SCA/SCAI/SIR/ STS/SVM guidelines for the diagnosis and management of patients with Thoracic Aortic Disease: a report of the American College of Cardiology Foundation/American Heart Association Task Force on Practice Guidelines, American Association for Thoracic Surgery, American College of Radiology, American Stroke Association, Society of Cardiovascular Anesthesiologists, Society for Cardiovascular Angiography and Interventions, Society of Interventional Radiology, Society of Thoracic Surgeons, and Society for Vascular Medicine. Circulation 2010; 121: e266-e369.

27. Nienaber C, Richartz B, Rehders T, Ince H, Petzsch M. Aortic intramural haematoma: natural history and predictive factors for complications. Heart 2004; 90: 372-4.

28. Evangelista A. Aortic intramural haematoma: remarks and conclusions. Heart 2004; 90: 379-80.

29. Golledge J, Eagle K. Acute aortic dissection. Lancet 2008; 372: 55-66.

30. Cañadas M, Vilacosta I, Ferreirós J, Bustos A, DíazMediavilla J, Rodríguez E. Hematoma intramural aórtico y anticoagulación. Rev Esp Cardiol 2007; 60: 201-4.

31. Soto J. Dolor Toráxico. Rev Med Clin Condes 2011; 22 (5): 655-64.

32. Glower D, Speier R, White W, Smith L, Rankin J, Wolfe W. Management and long-term outcome of aortic dissection. Ann Surg 1991; 214: 31-41.

33. Nallamothu B, Mehta R, Saint S, Llovet A, Bossone E, Cooper J, et al. Syncope in acute aortic dissection: diagnostic, prognostic, and clinical implications. Am J Med 2002; 113: 468-71.

34. Bossone E, Rampoldi V, Nienaber C, Trimarchi S, Ballotta A, Cooper J, et al. Usefulness of pulse deficit to predict in-hospital complications and mortality in patients with acute type A aortic dissection. Am J Cardiol 2002; 89: 851-5.

35. Januzzi J, Sabatine M, Eagle K, Evangelista A, Bruckman $\mathrm{D}$, Fattori R, et al. Iatrogenic aortic dissection. Am J Cardiol 2002; 89: 623-6.

36. Trimarchi S, Nienaber C, Rampoldi V, Myrmel T, Suzuki $\mathrm{T}$, Bossone $\mathrm{E}$, et al. Role and results of surgery in acute type B aortic dissection: insights from the International Registry of Acute Aortic Dissection(IRAD). Circulation 2006; 114: I357-64.

37. Laas J, Heinemann H, Schaefers H, Daniel W, Borst H. Management of thoracoabdominal malperfusion in aortic dissection. Circulation 1991; 84 Suppl III:III: 20-4.

38. Williams D, Lee D, Hamilton B, Marx M, Narasimham D, Kazanjian S, et al. The dissected aorta: part III. Anatomy and diagnosis of branch-vessel compromise. Radiology 1997; 203: 37-44.

39. Luker G, Glazer H, Eagar G, Gutiérrez F, Sagel S. Aortic dissection: effect of prospective chest radiographic diagnosis on delay to definitive diagnosis. Radiology 1994; 193: 813-9.

40. Jagannath A, Sos T, Lockhart S, Saddekni S, Sniderman K. Aortic dissection: a statistical analysis of the usefulness of plain chest radiographic findings. Am J Radiol 1986; 147: 1123-6.

41. Selman R, Kursbaum A, Ubilla M, Turner E, Espinoza C, Espinosa J, et al. Disección aórtica tipo A: Resultados operatorios y seguimiento a mediano plazo. Rev Med Chile 2010; 138: 982-7.

42. San Román J, Vilacosta I, Fernández Avilés F. Disección de aorta: evaluación clínica, comparación de las técnicas diagnósticas y elección del tratamiento. Rev Esp Cardiol 1996; 49 (Supl 4): 2-12.

43. Willoteaux S, Nedelcu C, Bouvier A, Hoareau J, Biere L, Thouveny F, et al. Acute aortic syndrome: which type of imaging to use? Presse Med 2011; 40 (1 Pt 1): 43-53.

44. Khan I, Nair C. Clinical, diagnostic, and management perspectives of aortic dissection. Chest 2002; 122: 31128.

45. Nienaber C, Kodolitsch Y, Petersen B, Loose R, Helmchen $\mathrm{U}$, Haverich A, et al. Intramural haemorrage of the thoracic aorta. Diagnostic and terapeutic implications. Circulation 1995; 92: 1465-72.

46. Evangelista A. Avances en el síndrome aórtico agudo. Rev Esp Cardiol 2007; 60: 428-39.

47. Ince $\mathrm{H}$, Nienaber C. Tratamiento de los sindromes aórticos agudos. Rev Esp Cardiol 2007; 60 (5): 526-41.

48. Erbel R, Alfonso F, Boileau C, Dirsch O, Eber B, Haverich A, et al. Diagnosis and management of aortic dissection. Eur Heart J 2001; 22: 1642-81. 
49. Rampoldi V, Trimarchi S, Eagle K, Nienaber C, Oh J, Bossone E, et al. Simple risk models to predict surgical mortality in acute type A aortic dissection: the International Registry of Acute Aortic Dissection score. Ann Thorac Surg 2007; 83: 55-61.

50. Gaul C, Dietrich W, Friedrich I, Sirch J, Erbguth F. Neurological symptoms in type A aortic dissections. Stroke 2007; 38: 292-97.

51. Centofanti P, Flocco R, Ceresa F, Attisani M, La Torre M, Weltert $\mathrm{L}$, et al. Is surgery always mandatory for type A aortic dissection? Ann Thorac Surg 2006; 82: 1658-63.

52. Santini F, Montalbano G, Casali G, Messina A, Iafrancesco M, Luciani G, et al. Clinical presentation is the main predictor of in-hospital death for patients with acute type A aortic dissection admitted for surgical treatment: a 25 years experience. Int J Cardiol 2007; 115: 305-11.

53. Tan M, Morshuis W, Dossche K, Kelder J, Waanders F, Schepens M. Long-term results after 27 years of surgical treatment of acute type a aortic dissection. Ann Thorac Surg 2005; 80: 523-9.

54. Dake M, Kato N, Mitchell R, Semba C, Razabi M, Shimono T, et al. Endovascular stent-graft placement for the treatment of acute aortic dissection. N Engl J Med 1999; 340: 1546-52.

55. Nienaber C, Fattori R, Lund G, Dieckmann C, Wolf W, von Kodolitsch $\mathrm{Y}$, et al. Non surgical reconstruction of thoracic aortic dissection by stent-graft placement. N Engl J Med 1999; 340: 1539-45.

56. Tsai T, Fattori R, Trimarchi S, Isselbacher E, Myrmel T,
Evangelista A, et al. Long-term survival in patients presenting with type B acute aortic dissection: insights from the International Registry of Acute Aortic Dissection. Circulation 2006; 114: 2226-31.

57. Karmy-Jones R, Simeone A, Meissner M, Granvall B, Nicholls S. Descending thoracic aortic dissections. Surg Clin North Am 2007; 87: 1047-8.

58. Nienaber C, Kische S, Zeller T, Rehders T, Schneider H, Lorenzen B, et al. Provisional extension to induce complete attachment after stent-graft placement in type B aortic dissection: the PETTICOAT concept. J Endovasc Ther 2006; 13 (6): 738-46.

59. Akin I, Kische S, Rehders T, Ince H, Nienaber C. Tratamiento en la disección aórtica torácica con prótesis endovascular. Rev Arg Cir Cardiovasc 2011; 9 (2): 10819.

60. Gilon D, Mehta R, Oh J, Januzzi J, Bossone E, Cooper $\mathrm{J}$, et al. Characteristics and in-hospital outcomes of patients with cardiac tamponade complicating type A acute aortic dissection. Am J Cardiol 2009; 103 (7): 1029.

61. Sebastià C, Pallisa E, Quiroga S, Alvarez-Castells A, Domingue R, Evangelista A. Aortic dissection: diagnosis and follow-up with helical CT. RadioGraphics 1999; 19: 45-60.

62. Braverman A. Acute aortic dissection: clinician update. Circulation 2010; 122: 184-8.

63. Juang D, Braverman A, Eagle K. Cardiology patient pages. Aortic dissection. Circulation 2008; 118: e507-10. 